International Journal of Clinical Dermatology \& Research (IJCDR)

ISSN 2332-2977

\title{
Vitiligo Associated With Pegylated Interferon and Ribavirin Treatment of Patients With Chronic Hepatitis C.
}

Review Article

Douhi $\mathrm{Z}^{1 *}$, Gallouj $\mathrm{S}^{1}$, Meziane $\mathrm{M}^{1}$, Aqoudad $\mathrm{N}^{2}$, Ibrahimi $\mathrm{A}^{2}$, Mernissi $\mathrm{Fz}^{1}$

${ }^{1}$ Department of dermatology, University Hospital Hassan II Fès, Morocco.

${ }^{2}$ Department of gastro-entérology, University Hospital Hassan II Fès, Morocco.

Abstract
Vitiligo is an autoimmune disease characterized by depigmented patches and macules. It is associated with many autoimmune diseases,
the most common of which is thyroid diseases. The association between Interferon (IFN) therapy and vitiligo is rarely reported in the
literature, despite its common usage in many diseases particularly hepatitis C viral infection (HCV). We report four cases of vitiligo that
appeared after treating HCV with pegylated IFN.

Keys words: hepatitis, Interferon, ribavirine, induced-vitiligo

\section{*Corresponding Author:}

Douhi. Z,

Department of dermatology,

University Hospital Hassan II Fès,

Morocco.

E-mail: drzakia1@hotmail.com

Received :July 03,2014

Accepted: August 15, 2014

Published: August 20, 2014

Citation: Douhi Z, et al (2014) Vitiligo Associated With Pegylated Interferon and Ribavirin Treatment of Patients With Chronic Hepatitis. C. Int J Clin Dermatol Res. 2(5), 27-29, doi: http://dx.doi. org $/ 10.19070 / 2332-2977-140008$

Copyright: Douhi. Z ${ }^{\odot} 2014$ This is an open-access article distributed under the terms of the Creative Commons Attribution License, which permits unrestricted use, distribution and reproduction in any medium, provided the original author and source are credited.

\section{Introduction}

The use of pegylated interferon (PEG-IFN) and ribavirin is considered standard therapy for patients with chronic hepatitis $\mathrm{C}$ (CHC) [1]. Many adverse effects of IFN appear to be of autoimmune origin; the literature contains few reports of vitiligo induced by IFN-alpha and rare cases associated with PEG-IFN. We report a series of four cases of vitiligo witch arising in the context of treatment for chronic hepatitis C.

\section{Materials and Methods}

A total of 58 patients were recruited in a prospective, monocentric study on dermatological side effects of treatment with Peg IFN and ribavirin in the HVC, for a period of one year. Among these patients, four cases had presented un induced vitiligo outside the injection site of PEG-INF (8,6\% of the total), with a median time to onset of twelve weeks.

Case 1: A 46 year-old female known to have diabetes mellitus and CHC under PEG-IFN 1,5 ug/ kg/week and ribavirin. Examina- tion revealed hypopigmented patches over the genital and anal area (Figures 1, 2). Wood's lamp examination showed contrast enhancement. There was no personal or family history of vitiligo, alopecia areata or thyroid disease. The patient was given betamethasone dipropionate $0.05 \%$ cream once a day for 3 months and the lesions resolved with minimal residual. IFN was withheld for 2 months and resumed again for an additional 10 months

Case 2: A 57 year-old male with CHC was treated with PEG-IFN and ribavirin and developed new onset vitiligo over the lip and genital area (Figures 3, 4) after 6 months of taking PEG-IFN and ribavirin. There was no personal or family history of vitiligo, alopecia or thyroid disease. He received topical Protopic for 2 months and he responded very well to treatment.

Case 3: A 54 year-old female known to have goiter and $\mathrm{CHC}$, developed ill-defined depigmented macules over the finger, after 1 months of initiating therapy. No treatment was given and his condition has not changed. There was no personal or family history of vitiligo.

Case 4: A 47 year-old male with CHC was treated with PEG-IFN and ribavirin and developed new onset vitiligo over the trunk after 4 months of initiating therapy (Figure 6). He received topical corticosteroids for 3 months and he responded well to treatment. There was no personal or family history of vitiligo, alopecia or thyroid disease.

\section{Discussion}

Hepatitis $\mathrm{C}$ virus (HCV) is a major public health problem worldwide. The use of PEG-IFN and ribavirin is the standard therapy for patients with $\mathrm{CHC}$ and both drugs may cause adverse effects [1].

PEG-IFN is a protein capable of inducing antiviral activity in cells. Pegylation of IFN has significantly changed pharmacologic properties of IFN: absorption is slowed (time for peak plasma concentration is multiplied by 7), clearance is reduced by about 10 times and the volume of distribution is less than 4 times. These 
Figures 1, 2: hypopigmented patches on the genital and anal area
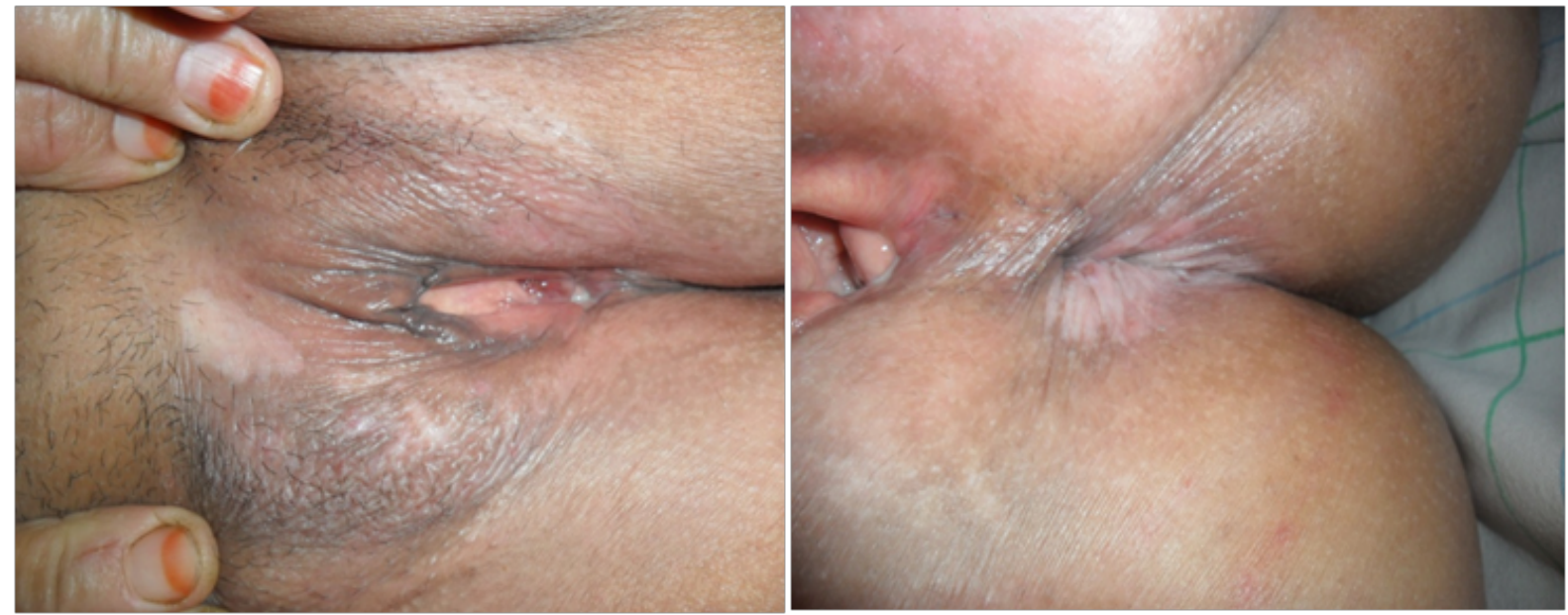

Figures 3, 4: hypopigmented patches over the lip and genital area

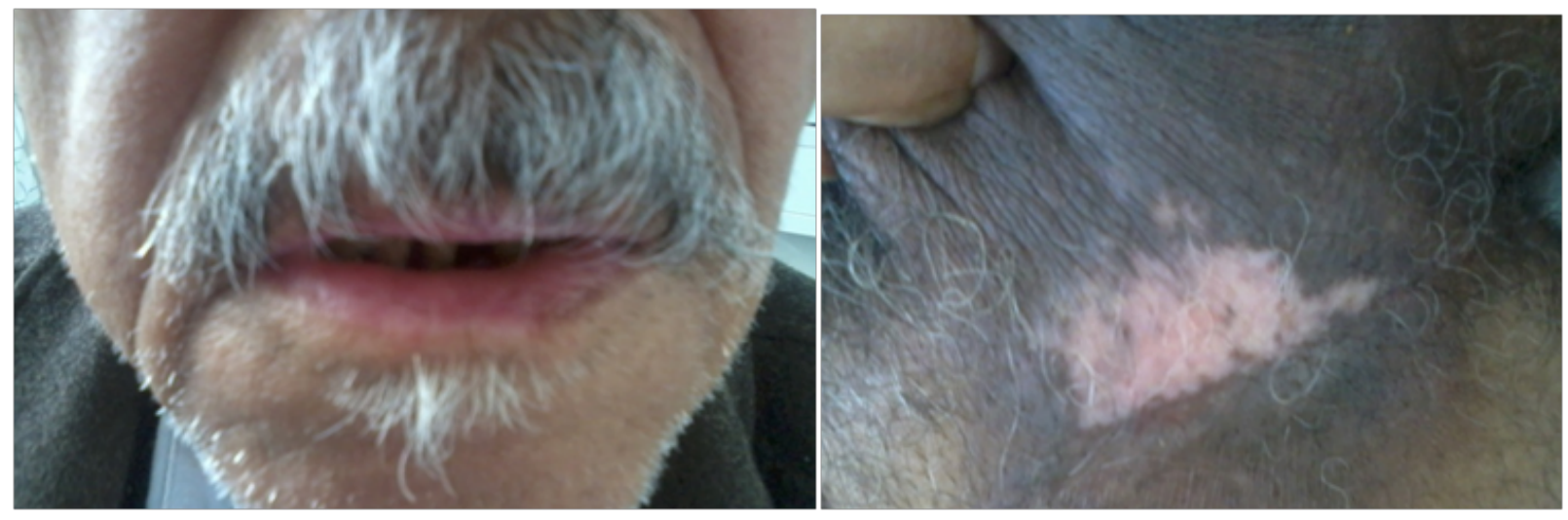

Figure 5. depigmented macules over the finger

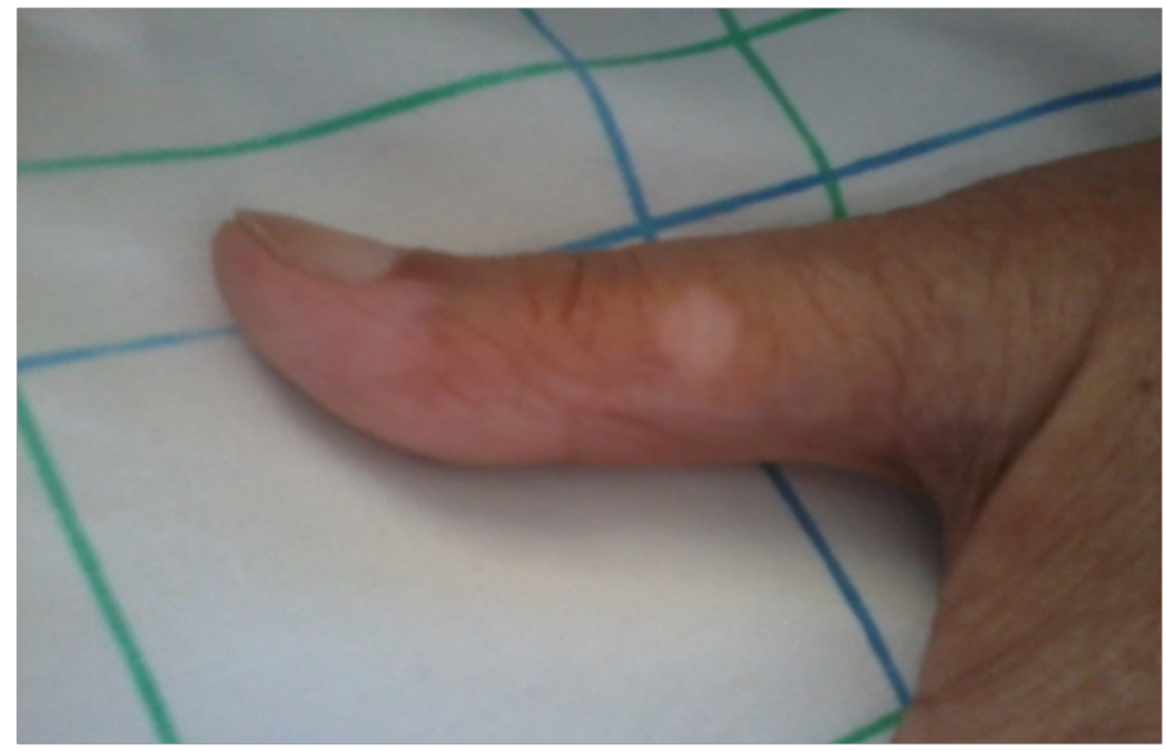


Figure 6. depigmented macules over the trunk

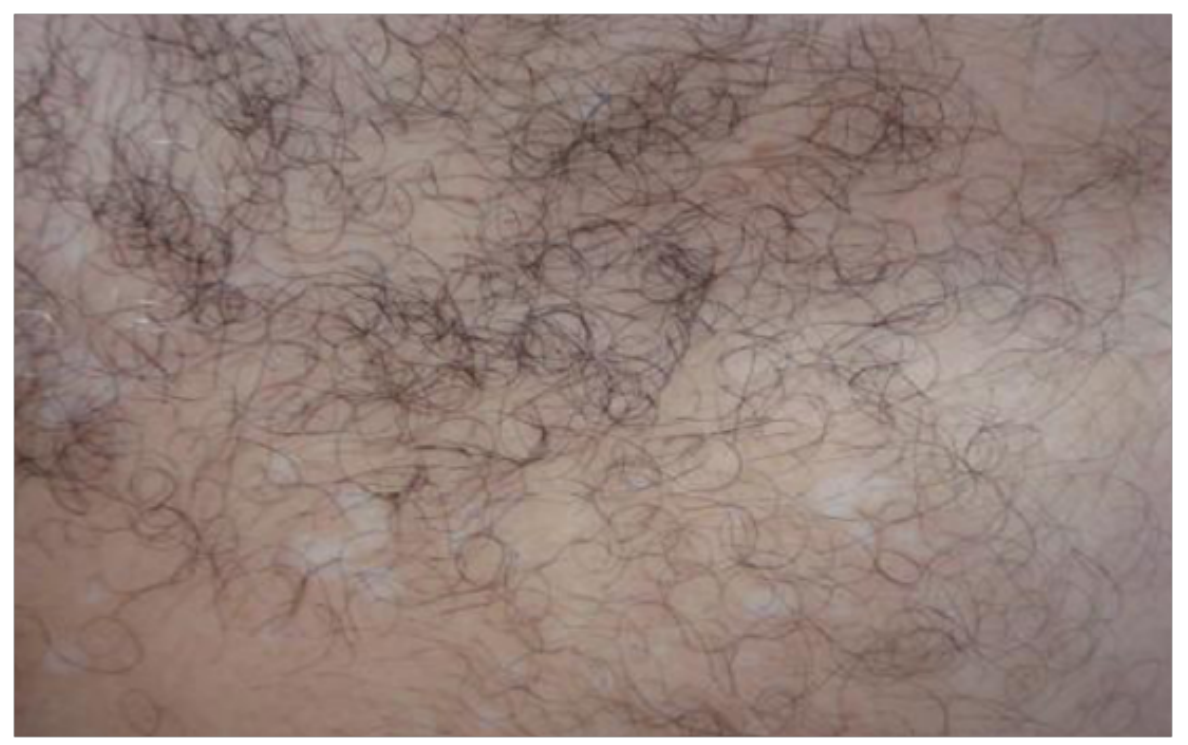

properties reduce the number of injections to once a week [2]. PEG-IFN side effects are dose dependent [3]. IFN-induced de rmatological side effects can be divided into immune related and nonimmune related. Immune mediated dermatological side effects include psoriasis, pemphigus, vitiligo, alopecia and systemic lupus erythematosus [4-5-6];

Occurrence of vitiligo in patients treated with pegylated IFN for viral hepatitis is rarely reported in the literature, being the subject of individual case reports. We herein report four cases arising in the context of treatment for chronic hepatitis C.

Vitiligo is an autoimmun disease characterized by depigmented patches and macules. It is associated with many autoimmune diseases. The association between IFN therapy and vitiligo is rarely reported in the literature, despite its common usage in hepatitis B viral infection, $\mathrm{HCV}$, hematological malignancies and melanoma [3-4].

Occurrence of vitiligo in patients with hepatitis under PEG-IFN therapy has been reported to occur as early as 1 month. The longest time interval between starting treatment and appearance of vitiligo is between 18 and 35 months [7]. However, in our series the shortest duration was 1 months, while the longest is 6 months. It occurs in the fourth to the sixth decade of life as clearly noticed in our series; and affects both genders equally.

The exact mechanism responsible for this autoimmune phenomenon is still unknown, but it most likely related to the biological features of IFN. Two hypotheses have been advanced: the presence of monoclonal antibodies directed against tyrosinase and melanocyte tyrosinase related protein; and activation of cytotoxic T lymphocytes with inhibition of suppressor $\mathrm{T}$ cell function [7-8].
Autoimmun adverse effects of IFN therapy, which may include vitiligo, should be carefully monitored. The decision to discontinue treatment must be discussed with the patient and based on the physician's clinical evaluation. The patient should be advised that skin lesions may persist [1].

\section{Conclusion}

The very low incidence of vitiligo attributable to the treatment with PEG-IFN suggests that IFN plays a role in unmasking vitiligo in susceptible individuals rather than causing it.

\section{References}

[1]. Krzysztof Tomasiewicz, Romana Modrzewska, Graz•yna Semczuk. Vitiligo Associated With Pegylated Interferon and Ribavirin Treatment of Patients With Chronic Hepatitis C: A Case Report. Advances in Therapy 2006 ; 23 : $139-142$.

[2]. Boyer N, Marcellin P. L'interféron pégylé : nouveau progrès dans letraitement de l'hépatite chronique virale C. Gastroenterol Clin Biol2000;24:767-769.

[3]. Raanani P, Ben-Bassat I. Immune-mediated complications during interferon therapy in hematological patients. Acta Haematol 2002; 107:133-144.

[4]. Sacchi S, Kantarjian H, O’Brien S, et al. Immunemediated and unusual complications during interferon alfa therapy in chronic myelogenous leukemia. J Clin Oncol 1995; 13: 2401-2407.

[5]. Asnis LA, Gaspari AA. Cutaneous reactions to recombinant cytokines therapy. J Am Acad Dermatol 1995; 33: 393-410.

[6]. Tinio P, Hadi A, Al-Ghaithi K, et al. Segmental vitiligo and hair curling after interferon alpha and ribavirin treatment for hepatitis C. Skinmed 2006; 5: 50-51.

[7]. Hamadah I, Binamer Y, Sanai FM, Abdo AA, Alajlan A. Interferoninducedvitiligo in hepatitis C patients: a case series. In J Dermatol 2010, 49, 829-833.

[8]. Simsek H, Savas C, Akkiz H, Telatar H. Interferon induced vitiligoin a patient with chronic viral hepatitis C infection. Dermatology 1996;193: 65-66. 